\title{
Adaptive regression modeling of biomarkers of potential harm in a population of U.S. adult cigarette smokers and nonsmokers
}

\author{
John H Warner ${ }^{1 \dagger}$, Qiwei Liang ${ }^{2 *}$, Mohamadi Sarkar², Paul E Mendes ${ }^{2}$, Hans J Roethig ${ }^{2}$
}

\begin{abstract}
Background: This article describes the data mining analysis of a clinical exposure study of 3585 adult smokers and 1077 nonsmokers. The analysis focused on developing models for four biomarkers of potential harm (BOPH): white blood cell count (WBC), 24 h urine 8-epi-prostaglandin $F_{2 \alpha}$ (EPI8), 24 h urine 11-dehydro-thromboxane $B_{2}(D E H 11)$, and high-density lipoprotein cholesterol (HDL).

Methods: Random Forest was used for initial variable selection and Multivariate Adaptive Regression Spline was used for developing the final statistical models

Results: The analysis resulted in the generation of models that predict each of the BOPH as function of selected variables from the smokers and nonsmokers. The statistically significant variables in the models were: platelet count, hemoglobin, C-reactive protein, triglycerides, race and biomarkers of exposure to cigarette smoke for WBC (R-squared $=0.29)$; creatinine clearance, liver enzymes, weight, vitamin use and biomarkers of exposure for EPI8 (R-squared $=0.41)$; creatinine clearance, urine creatinine excretion, liver enzymes, use of Non-steroidal antiinflammatory drugs, vitamins and biomarkers of exposure for DEH11 (R-squared $=0.29$ ); and triglycerides, weight, age, sex, alcohol consumption and biomarkers of exposure for HDL (R-squared $=0.39$ ).

Conclusions: Levels of WBC, EPI8, DEH11 and HDL were statistically associated with biomarkers of exposure to cigarette smoking and demographics and life style factors. All of the predictors togather explain $29 \%-41 \%$ of the variability in the $\mathrm{BOPH}$.
\end{abstract}

\section{Background}

Cigarette smoking is a well known risk factor for cardiovascular diseases [1]. Commonly accepted pathophysiological mechanisms underlying many cigarette smoking associated adverse health effects are inflammation [2,3], oxidative stress $[2,4]$, platelet activation $[5,6]$ and abnormal lipid metabolism $[7,8]$. Suitable biomarkers of potential harm (BOPH) have been identified for these four different pathophysiological pathways: white blood cell counts (WBC) for inflammation $[3,9,10]$, urine 8epi-prostaglandin $\mathrm{F}_{2 \alpha}$ (EPI8) for oxidative stress [11-13], urine 11-dehydro-thromboxane B2 (DEH11) for platelet activation $[11,13,14]$, and high-density lipoprotein cholesterol (HDL) for abnormal lipid metabolism [15].

\footnotetext{
* Correspondence: qiwei.liang@altria.com

+ Contributed equally

${ }^{2}$ Altria Client Services Inc, 601 E Jackson Street, Richmond, VA 23219, USA
}

The Total Exposure Study (TES) was a stratified, cross-sectional, multi-center study in 3585 adult smokers and 1077 nonsmokers, designed with the primary objective of estimating the exposure to cigarette smoke constituents in a population of U.S. adult cigarette smokers [16]. A secondary objective of the study was to investigate the relationship between cigarette smoke exposure and biomarkers of potential harm. The purpose of this study was to explore relationships between the variables in the TES and four biomarkers of potential harm and to capture those relationships in statistical models.

\section{Methods}

The TES study database contains data on biomarkers of potential harm, biomarkers of exposure (BOE), smoking history, medical history, concomitant medications, clinical laboratory results, and demographics for 3585 adult 
smokers and 1077 non-smokers. Details about the study have been previously reported [16,17]. The biomarkers of exposure included nicotine equivalents (NICEQ), serum cotinine (COTIN), 4-(methylnitrosamino)-1-(3pyridyl)-1-butanol (NNAL) and NNAL glucuronides (TOTNN), carboxyhemoglobin $(\mathrm{COHb})$, monohydroxybutenyl-mercapturic acid (MHBMA), mercapturic acid metabolites dihydroxy-butyl-mercapturic acid (DHBMA), 4-aminobiphenyl (4-ABP) hemoglobin adducts, 1-hydroxypyrene (1-OHP) and 3-hydroxypropylmercapturic acid (3-HPMA). These biomarkers are indicators of exposure to cigarette smoke and represent cigarette smoke constituents. Details about the biomarkers and the smoke constituents represented by these biomarkers can be found in Roethig et al. [16]. They were measured in either urinary samples or blood samples in the TES study $[16,17]$.

\section{Overview of variables in the data mining database}

In the data mining, variables were selected based on their scientific relevance to the targeted biomarkers of potential harm. Table 1 provides an overview of the variables that appear in the data mining datasets and Table 2 has the full names of the biomarkers of potential harm.

Separate imputed data mining data sets were constructed for each BOPH. In these data sets, cases were dropped if the value of the dependant variable was missing; values for predictor variables were imputed using methods that are described below. In addition, an unimputed data mining data set was constructed. No cases were dropped from the unimputed data sets and no imputation of missing values was performed on it. The data mining data sets were randomly divided into analysis and validation data sets using an $80 / 20$ split.

\section{Data mining analyses using random forests}

The Random Forest procedure [18-20], a data mining approach for variable selection and model building, was used to perform a preliminary screening of variables for each BOPH (DEH11, EPI8, HDL and WBC). The R statistical package [21] was used for the implementation of Random Forest. Variables that were known to be trivially related to the target $\mathrm{BOPH}$ were not included in the initial or subsequent Random Forest runs. In the initial Random Forest analyses, 10,000 trees were generated, and variable importance and cross-validated R-squared statistics were produced. The variable importance effectively ranks all variables in each data set with respect to their ability to predict the target BOPH. At the end, 30 predictor-sets together with cross-validated R-squared statistics were kept. From this list, a large, a medium, and a small predictor set was chosen for input into the Multivariate Adaptive Regression Spline (MARS) procedure [22]. In each case, the large predictor set was chosen to contain 30 variables, while medium and small data sets were chosen to represent natural cut points in the sequence of cross-validated R-squared values.

\section{Data mining analyses using MARS}

Starting with the large, medium and small predictor sets selected by the Random Forests procedure, the MARS algorithm [23] was used to find interpretable models for each BOPH (DEH11, EPI08, HDL, WBC) in each study population (all subjects, non-smokers, smokers). MARS is an innovative and flexible modeling approach that uncovers important data patterns and relationships. It builds flexible models by the use of separate regression slopes in distinct intervals of the predictor variable space. This approach has been increasingly used in recent years in various scientific fields including disease risk research [24,25], human genetics [26] and food sciences [27].

\begin{tabular}{|c|c|}
\hline Group & Variables \\
\hline $\mathrm{BOPH}(4)$ & DEH11, EPI8, HDL, WBC \\
\hline $\begin{array}{l}\text { Special interest variables: } \\
\text { BOE (9): }\end{array}$ & $\begin{array}{l}\text { HPMA3, DHBMA, MHBMA, NICEQ, COTIN, } \\
\text { OHP, TOTNN, ABP, COHB }\end{array}$ \\
\hline $\begin{array}{l}\text { Special interest variables: } \\
\text { cumulative effects ( } 2 \text { ) }\end{array}$ & $\begin{array}{l}\text { "AGE", "SMKYRS" (years smoked, excluded } \\
\text { from analysis of non-smokers) }\end{array}$ \\
\hline $\begin{array}{l}\text { Special interest variables: } \\
\text { stratification (1) }\end{array}$ & Smoking Status \\
\hline Exposure variables (4) & $\begin{array}{l}\text { Measures of exposure to exhaust and } \\
\text { chemicals, from questionnaire }\end{array}$ \\
\hline $\begin{array}{l}\text { Exposure variables } \\
\text { (non-smokers, 8) }\end{array}$ & $\begin{array}{l}\text { Measures of exposure to secondary } \\
\text { smoke, from questionnaire }\end{array}$ \\
\hline $\begin{array}{l}\text { Exposure variables } \\
\text { (smokers, 19) }\end{array}$ & $\begin{array}{l}\text { Measures of exposure to tobacco, } \\
\text { including number of cigarettes smoked, } \\
\text { tar and nicotine content, presence of } \\
\text { menthol, included only in the analysis for } \\
\text { smokers }\end{array}$ \\
\hline Demographics (13) & $\begin{array}{l}\text { Weight, gender, race, geographical } \\
\text { location, income, etc. }\end{array}$ \\
\hline Vital signs (5) & $\begin{array}{l}\text { Respiratory rate, temperature, blood } \\
\text { pressure, pulse }\end{array}$ \\
\hline Clinical measures (2) & Measures of respiratory capacity, FVC, FEV \\
\hline General health (20) & $\begin{array}{l}\text { General health questions, from } \\
\text { questionnaire }\end{array}$ \\
\hline Lab values (22) & Clinical chemistry laboratory values \\
\hline Creatinine clearance (1) & $\begin{array}{l}\text { CRCL: } 24 \text { h urine creatinine/plasma } \\
\text { creatinine }\end{array}$ \\
\hline Lab value flags (6) & Lab value flags \\
\hline $\begin{array}{l}\text { Medical history indicators } \\
\text { (15) }\end{array}$ & $\begin{array}{l}\text { Medical history findings broken down into } \\
15 \text { categories }\end{array}$ \\
\hline $\begin{array}{l}\text { Concomitant medications } \\
\text { (61) }\end{array}$ & $\begin{array}{l}\text { Concomitant medications broken down } \\
\text { into } 61 \text { categories }\end{array}$ \\
\hline
\end{tabular}


Table 2 Variables appearing in the final models, names and abbreviations

\begin{tabular}{|c|c|c|}
\hline Names & Abbreviations & Units/category names \\
\hline $\begin{array}{l}24 \mathrm{~h} \text { urine 11-Dehydro- } \\
\text { thromboxane } \mathrm{B}_{2}\end{array}$ & DEH11 & ng/24 hr urine \\
\hline $\begin{array}{l}24 \text { h urine } 8 \text {-epi- } \\
\text { prostaglandin } \mathrm{F}_{2 \alpha}\end{array}$ & EPI8 & $\mathrm{ng} / 24 \mathrm{hr}$ urine \\
\hline $\begin{array}{l}\text { High density lipoprotein } \\
\text { cholesterol }\end{array}$ & $\mathrm{HDL}$ & $\mathrm{ng} / \mathrm{dL}$ \\
\hline White Blood Cell Count & WBC & $\times 10^{\wedge} 3 / \mathrm{uL}$ \\
\hline $\begin{array}{l}24 \mathrm{~h} \text { urine Nicotine } \\
\text { equivalents }\end{array}$ & NICEQ & $\mathrm{mg} / 24 \mathrm{hr}$ urine \\
\hline Serum Cotinine & COTIN & $\mathrm{ng} / \mathrm{mL}$ \\
\hline $\begin{array}{l}24 \mathrm{~h} \text { urine total } \\
1 \text {-hydroxypyrene }\end{array}$ & $\mathrm{OHP}$ & ng/24 hr urine \\
\hline $\begin{array}{l}24 \mathrm{~h} \text { urine total } \\
4 \text {-(methylnitrosamino)-1- } \\
\text { (3-pyridyl)-1-butanol (total } \\
\text { NNAL) }\end{array}$ & TOTNN & $\mathrm{ng} / 24 \mathrm{hr}$ urine \\
\hline Creatinine clearance & $\mathrm{CRCL}$ & $\mathrm{dL} /$ day \\
\hline $24 \mathrm{~h}$ urine creatinine & UCRCAL & $\mathrm{mg} / 24 \mathrm{hr}$ urine \\
\hline Aspartate aminotransferase & AST & $U / L$ \\
\hline Alkaline phosphatase & ALKPH & $\mathrm{U} / \mathrm{L}$ \\
\hline Serum Triglycerides & TRIG & $\mathrm{mg} / \mathrm{dL}$ \\
\hline Platelet count & PLATE & $\times 10^{\wedge} 3 / \mathrm{uL}$ \\
\hline $\begin{array}{l}\text { High-sensitivity C-reactive } \\
\text { protein }\end{array}$ & CRP & $\mathrm{mg} / \mathrm{L}$ \\
\hline Hemoglobin & $\mathrm{HGB}$ & $\mathrm{g} / \mathrm{dL}$ \\
\hline Age & AGE & Yrs \\
\hline Weight & WEIGHTK & $\mathrm{kg}$ \\
\hline Vitamin supplements & VITIMINS & yes, no \\
\hline Alcohol consumption & DRINK & $\begin{array}{l}>=\text { once/day, >once/ } \\
\text { week, once/week, } \\
<\text { once/week, no }\end{array}$ \\
\hline $\begin{array}{l}\text { Non-steroidal } \\
\text { antiinflammatory drugs }\end{array}$ & NSAID & no, yes \\
\hline Sex & SEX & "female", "male" \\
\hline Race & RACE & $\begin{array}{l}\text { other, native Amer, } \\
\text { multi-racial, Caucasian, } \\
\text { Asian, black }\end{array}$ \\
\hline If greater than & IFGT & \\
\hline If less than & IFLT & \\
\hline Equal & EQ & \\
\hline
\end{tabular}

In our study, the large, medium, and small predictor variable sets were augmented by adding all nine $\mathrm{BOE}$ and a binary indicator of smoking status. Finally, when ordinal categorical variables appeared in any of the predictor sets, they were replaced with appropriately chosen ordinal dummy variables. Starting with the modified large, medium and small predictor sets, exploratory MARS models were fit for a variety of model types, settings for the number of initial basis functions and penalties for adding new variables.

In order to evaluate the appropriateness of the model, MARS uses generalized cross-validation (GCV) which is residual square errors penalized by a function related to complexity of the model [22]. The numerator in GCV is the average residual squared error and the denominator is a penalty term that reflects model complexity. The use of the denominator is to prohibit selection of a model with many terms that decreases only slightly the residual errors. The GCV statistic is an estimate of the variance for error in a regression model that includes a penalty term for the number of parameters used in the regression. The GCV R-squared statistic is the ordinary R-squared statistic calculated with the variance for error replaced with the GCV statistic [22].

Final MARS runs were performed using exact variable searches $($ SPEED $=1)$, three model types $(0=$ "simple linear model", 1 = "linear model with variable transformations but no interactions", and $2=$ "two-way interaction models with variable transformations"), 4 values for the number of initial basis functions $(10,20,30$, and 60 ), and 2 values for a penalty term for adding new variables ( 0 , and .01). As before, a 10-fold cross-validation was used to estimate the cross-validated (or GCV) Rsquared statistic. For each BOPH and predictor set, GCV R-squared, the number of final model parameters, and the number of final model variables were plotted against all other MARS control parameters. These plots (not included in this manuscript) were examined and a preferred model was selected for each $\mathrm{BOPH}$ and each study population. Models were preferred if they fit the data well (i.e. had high GCV-R-squared values) and parsimonious (i.e. had few predictors).

Using the imputed analysis data sets the MARS procedure was used to fit models to each of the four BOPH using large, medium, and small predictor sets obtained in Random Forests. This procedure produced closedform linear regression models that display explanatory power comparable to the best Random Forest models. For each of the four BOPH, a preferred MARS model was selected from the MARS runs based on considerations of goodness of fit and parsimony. The selected models were applied and refit in S-PLUS using the validation data set [28]. Graphical model displays are presented to aid in model interpretation. Applying and refitting models using the validation data set protects against model over-fit that can result from aggressive use of data mining procedures.

Variable importance plots were provided for each $\mathrm{BOPH}$. The marginal $\mathrm{R}$-squared value for a group of predictor variables is the proportion of variance of the dependent variable explained by the group of predictor variables. The delta $\mathrm{R}$-squared for a group of predictor variables is the difference between the marginal $R$ squared value for the full set of dependent variables and the marginal $\mathrm{R}$-squared value for the full set of predictor variables with the specified group removed. The 
marginal R-squared is a measure of the quality of prediction obtained from a group of predictor variables acting alone, and the delta R-square is a measure of the improvement in the quality of prediction obtained when a group of predictor variables is added to the other predictor variables in the model.

\section{Handling of missing data and BLLOQ values}

In general, missing data were present in the data set, but rare. In the data mining analysis, it was deemed prudent to impute missing data. Missing continuous variables were imputed in MARS and Random Forest Analyses by replacing missing observations with the median of nonmissing cases for each variable: this was done separately for smokers and non-smokers, and for validation and analysis datasets. Imputation was carried out on predictor variables only, cases with missing dependant variables were dropped, not imputed, in the MARS and Random Forest analyses.

Final regression models were fit to data sets in which no imputation was used on any variable, i.e. cases with missing dependant or predictor variables were dropped from the analysis and the number of cases dropped was noted in the regression output. In general, final regression results seemed little changed between imputed and unimputed datasets.

For categorical variables, missing data were handled by adding an additional category as indicated in the following section. This approach does not depend on any assumptions about the sources of missing data and we do not regard it as a form of imputation.

Below the lower limit of quantification (BLLOQ) flags were present in the data set for 9 variables of exposure (NECEQ, MHBMA, TOTNN, DHBMA, OHP, HPMA3, COTIN, COHB, and 4-ABP). If a BLLOQ flag is positive for any of these variables and if the variable itself has a missing value, the value of the affected variable is set to 0 . Non-missing value variables BLLOQ values with flags were left unchanged.

\section{Variable transformations}

The Random Forest and the MARS procedure are both designed to find optimal transformations of predictor variables. This minimized the need to perform variable transformations manually. Nonetheless a number of variable transformations were applied to a number of variables. In particular, character variables were transformed to dummy variable. A variable for creatinine clearance was also created. Initial random forest runs found some categorical variables that had natural ordinal interpretations: these variables were replaced with sets of ordinal dummy variables in the MARS and final regression analyses.

\section{Variable names and naming conventions for the final models}

Table 2 presents descriptive names and abbreviated names for all untransformed variables that appear in at least one of the final models. Units (for continuous variable) and category names (for categorical variables), are also provided.

Variable names in final regression models will be of one of the following forms

1. <V.name $>$

2. <v.name>.EQ.xxx, <v.name>.GT.xxx, <v.name>.

LT.xxx, <v.name>.GE.xxx, <v.name>.LE.xxx

3. <v.name>.IFLT.xxx, <v.name>.IFGT.xxx

Here, $<$ v.name $>$ is the short name of a variable from Table 2 and $\mathrm{xxx}$ is a number or category selected by MARS. Form 1 is used for variables that enter into the MARS models as linear functions. Form 2 is used to describe dummy variables formed from a categorical variable. In this context "EQ", "GT", "LT", "GE", "LE" stand for "equal", "greater than", "less than", "greater than or equal to", and "less than or equal to". For example, Race.EQ.BLACK designates a variable that is one for blacks and zero otherwise. Alcohol consumption. GT.1 PER WEEK designates a variable that is one for subjects who have more than one drink per week and 0 otherwise. Form 3 is used for transformed continuous variables. For example, CRCL.IFGT.100 (read CRCL if greater than 100) indicates a variable that is equal to CRCL if CRCL is greater than 100 and 100 otherwise. Similarly, CRCL.IFLT.100 is a variable that is equal to CRCL when CRCL is $<100$ and equal to 100 otherwise.

Using this notation, regression coefficients can be interpreted as slopes. Positive slopes indicate that the dependent variable increases as the predictor variable increases and negative slopes indicate that the dependant variable decreases as the predictor variable increases. Variables of Form 3 are called transformations of the original variable and are thought of as the result of applying a function (or transformation) to the original variable. Other commonly used transformations that do not appear in our regression output are square root, power, and log transformations.

Collectively, interactions and variables of forms 1,2 , and 3 are called basis functions. The MARS procedure, to be discussed below, may be thought of as a procedure for selecting an optimal set of basis functions that are to be used as the predictor of a given dependent variable. The basis functions used by MARS differ slightly from the basis functions described here. We have chosen to use the basis functions as described here in preference to those used by MARS, because the former seem easier to interpret. 


\section{Results}

Tables 3 and 4 present parameter estimates, standard errors and $\mathrm{p}$ values for the final models for each of the four $\mathrm{BOPH}$ for the analysis data set. The parameters often characterize multiple transformations of the same underlying predictor. Figures 1 to 2 show the importance plots of the predictor variables based on Marginal $\mathrm{R}$-squared and Delta R-squared values. All plots were based on unimputed data.

The model for DEH11 contains predictors COTIN, UCRCAL, CRCL, AST, ALKPH, NSAID, and VITAMIN (Table 3). The model accounts for $29 \%$ of the total variability in DEH11 in all subjects. Higher serum cotinine, urine creatinine and creatinine clearance were predictors of higher 24-hour urinary excretion of 11dehydrothromboxane B2. Higher serum AST (up to 126 $\mathrm{U} / \mathrm{L}$ ) was mainly a predictor of higher 24-hour urinary excretion of 11-dehydrothromboxane B2 while use of nonsteroidal anti-inflammatory agents and vitamin supplements were predictors of lower 24-hour urinary

Table 3 Parameter estimates in models for DEH11 and EPI8 for all subjects (analysis data set)

\begin{tabular}{lcccc}
\hline a) Model for DEH11 & \multicolumn{5}{c}{$\mathbf{R}^{\mathbf{2}}=\mathbf{0 . 2 9}$} \\
\hline Parameters & Value. & Std Error & $\mathbf{t}$ value & P value \\
\hline Intercept & -4045.3022 & 752.258 & -5.38 & $<0.001$ \\
\hline COTIN.IFGT.11 & 1.4494 & 0.123 & 11.78 & $<0.001$ \\
\hline UCRCAL.IFLT.3036 & 0.3531 & 0.0484 & 7.30 & $<0.001$ \\
\hline CRCL.IFLT.4325 & 0.3698 & 0.0391 & 9.45 & $<0.001$ \\
\hline AST.IFGT.25 & 17.9064 & 1.2832 & 13.95 & $<0.001$ \\
\hline AST.IFGT.126 & -18.2428 & 2.3656 & -7.71 & $<0.001$ \\
\hline ALKPH.IFLT.184 & 3.1360 & 0.6583 & 4.76 & $<0.001$ \\
\hline ALKPH.IFGT.184 & 30.7979 & 3.6627 & 8.41 & $<0.001$ \\
\hline NSAID.EQ.YES & -346.0560 & 38.2528 & -9.05 & $<0.001$ \\
\hline VITAMIN.EQ.YES & -211.7183 & 29.8500 & -7.09 & $<0.001$ \\
\hline
\end{tabular}

\begin{tabular}{lcccc}
\hline b) Model for EPI8 & \multicolumn{5}{c}{$\mathbf{R}^{2}=\mathbf{0 . 4 1}$} \\
\hline Parameters & Value. & Std Error & $\mathbf{t}$ value & P value \\
\hline Intercept & 1383.0200 & 574.483 & 2.41 & 0.016 \\
\hline TOTNN.IFLT.57 & 5.1813 & 0.7683 & 6.74 & $<0.001$ \\
\hline TOTNN.IFGT.57 & 0.5548 & 0.0593 & 9.35 & $<0.001$ \\
\hline TOTNN.IFGT.1452 & -1.4595 & 0.3895 & -3.75 & 0.000 \\
\hline OHP.IFLT.473 & 0.8552 & 0.1212 & 7.05 & $<0.001$ \\
\hline CRCL & 0.6268 & 0.0262 & 23.93 & $<0.001$ \\
\hline AST.IFGT.22 & -135.4800 & 30.5048 & -4.44 & $<0.001$ \\
\hline AST.IFGT.24 & 247.4960 & 59.5308 & 4.16 & $<0.001$ \\
\hline AST.IFGT.26 & -108.4000 & 33.1568 & -3.27 & 0.001 \\
\hline AST.IFLT.106 & 12.2512 & 2.2425 & 5.46 & $<0.001$ \\
\hline WEIGHTK & 6.2845 & 0.7274 & 8.64 & $<0.001$ \\
\hline VITAMIN.EQ.YES & -250.7700 & 28.4736 & -8.81 & $<0.001$ \\
\hline
\end{tabular}

Table 4 Parameter estimates in models for WBC and HDL for all subjects (analysis data set)

\begin{tabular}{lcccc}
\hline a) Model for WBC & \multicolumn{4}{c}{$\mathbf{R}^{\mathbf{2}}=\mathbf{0 . 2 9}$} \\
\hline Parameters & Value & Std. Error & $\mathbf{t}$ value & $\mathbf{P}$ value \\
\hline Intercept & -6.4951 & 1.4436 & -4.50 & $<0.001$ \\
\hline TOTNN.IFGT.51 & -0.0055 & 0.0026 & -2.17 & 0.030 \\
\hline TOTNN.IFLT.471 & 0.0078 & 0.0023 & 3.36 & 0.001 \\
\hline TOTNN.IFGT.471 & 0.0065 & 0.0026 & 2.49 & 0.013 \\
\hline CRP.IFLT.2 & 0.3426 & 0.0548 & 6.25 & $<0.001$ \\
\hline CRP.IFGT.2 & 0.0715 & 0.0096 & 7.48 & $<0.001$ \\
\hline CRP.IFGT.20 & -0.0477 & 0.0169 & -2.82 & 0.005 \\
\hline PLATE.IFLT.245 & 0.0106 & 0.0014 & 7.69 & $<0.001$ \\
\hline PLATE.IFGT.245 & 0.007 & 0.0007 & 10.64 & $<0.001$ \\
\hline HGB.IFLT.14 & 0.3242 & 0.0477 & 6.80 & $<0.001$ \\
\hline TRIG.IFLT.171 & 0.0051 & 0.0008 & 6.32 & $<0.001$ \\
\hline TRIG.IFGT.503 & 0.0025 & 0.0008 & 3.17 & 0.002 \\
\hline RACE.EQ.BLACK & -0.679 & 0.0914 & -7.42 & $<0.001$ \\
\hline
\end{tabular}

\begin{tabular}{lcccc}
\hline b) Model for HDL & \multicolumn{4}{c}{$\mathbf{R}^{2}=\mathbf{0 . 3 9}$} \\
\hline Parameters & Value & Std. Error & $\mathbf{t}$ value & P value \\
\hline Intercept & 55.4292 & 2.948 & 18.80 & $<0.001$ \\
\hline COTIN.IFGT.31 & -0.0291 & 0.0036 & -7.97 & $<0.001$ \\
\hline COTIN.IFGT.193 & 0.0258 & 0.0069 & 3.75 & 0.000 \\
\hline TRIG.IFGT.52 & -0.5819 & 0.0786 & -7.40 & $<0.001$ \\
\hline TRIG.IFGT.64 & 0.483 & 0.0816 & 5.92 & $<0.001$ \\
\hline TRIG.IFGT.177 & 0.0753 & 0.0086 & 8.76 & $<0.001$ \\
\hline WEIGHT.IFLT.76 & -0.267 & 0.032 & -8.34 & $<0.001$ \\
\hline WEIGHT.IFGT.76 & -0.0834 & 0.0157 & -5.30 & $<0.001$ \\
\hline AGE.IFLT.55 & 0.3119 & 0.0197 & 15.83 & $<0.001$ \\
\hline SEX.EQ.F & 6.72 & 0.4723 & 14.23 & $<0.001$ \\
\hline DRINK.EQ.Y & 2.0567 & 0.5099 & 4.03 & 0.0001 \\
\hline DRINK.GT.1.PER.WK & 7.875 & 0.5393 & 14.60 & $<0.001$ \\
\hline
\end{tabular}

excretion of 11-dehydrothromboxane B2. In the validation data set the same predictors were significant with the exception of ALKPH.

The model for EPI8 contains predictors TOTNN, OHP, CRCL, AST, WEIGHTK, and VITAMIN and it accounts for $41 \%$ of the total variability in EPI8 in all subjects (Table 3). Higher total NNAL (up to $1452 \mathrm{ng} /$ $24 \mathrm{~h}$ ), OHP (if less than $473 \mathrm{ng} / 24 \mathrm{~h}$ ), creatinine clearance and weight were predictors of higher 24-hour excretion of urinary 8-epi-Prostaglandinf $F_{2 \alpha}$ Type III. Higher serum AST was either a predictor of higher or lower 24-hour excretion of urinary 8-epi-Prostaglan$\operatorname{dinF}_{2 \alpha}$ Type III, depending on its concentration level. Use of vitamin supplements was a predictor of lower 24-hour excretion of urinary 8-epi-Prostaglandinf $F_{2 \alpha}$ Type III. In the validation data set the same predictors were significant with the exception of AST. 


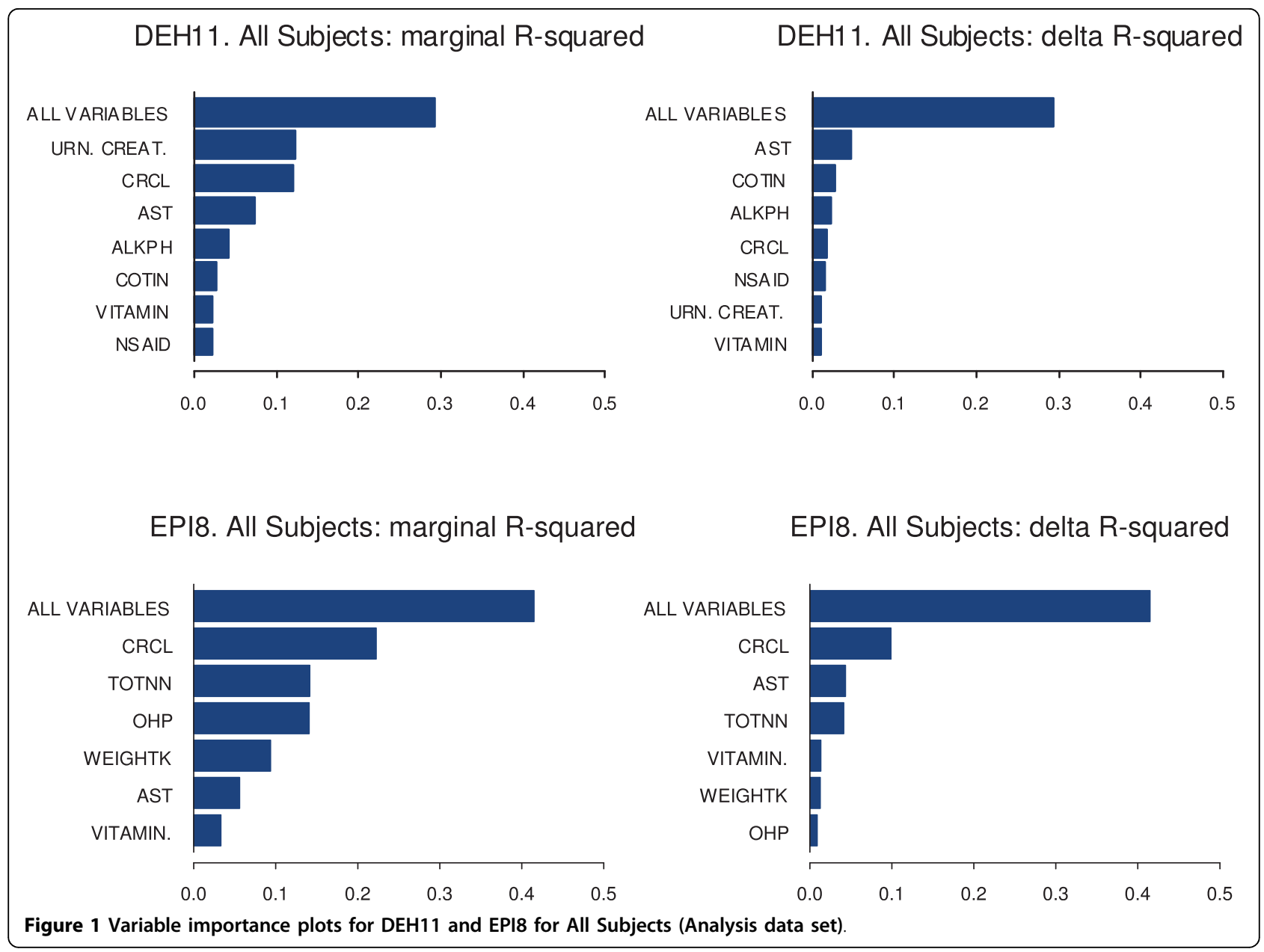

The model for WBC in all subjects contains predictors TOTNN, CRP, PLATE, HGB, TRIG, and RACE (Table 4). The model accounts for $29 \%$ of the total variability in WBC. Increases in total NNAL (if greater than 51 and less than $471 \mathrm{ng} / 24 \mathrm{~h}$ ), high sensitivity c-reactive protein (up to $20 \mathrm{mg} / \mathrm{L}$ ), platelet count, hemoglobin (if less than $14 \mathrm{~g} / \mathrm{DL}$ ), and triglycerides were predictors of higher WBC count, while race categories of Black and "other" were predictors of lower WBC count. In the validation data set the same predictors were significant.

The model for HDL in all subjects contains predictors COTIN, TRIG, WEIGHTK, HGB, AGE, SEX, and ALCOHOL CONSUMPTION (Table 4). The model accounts for $39 \%$ of the total variability in HDL. Higher age, alcohol consumption and female gender were predictors of higher HDL cholesterol, while higher triglycerides was a predictor of lower HDL cholesterol. Higher weight and cotinine (if less than $31 \mathrm{ng} / \mathrm{ml}$ ) were predictors of lower HDL cholesterol. In the validation data set the same predictors were significant.

Table 5 provides a summary of fit statistics across models for the analysis data set and the validation data set. Figures 1 and 2 gives the importance plots for the predictors for the $4 \mathrm{BOPH}$.

\section{Discussion}

The primary purpose of this analysis was to explore the quantitative associations of biomarkers of exposure and other variables with biomarkers of potential harm related to cigarette smoking. We examined the covariates (or secondary variables) to see whether they could mediate or modulate the effect of biomarkers of exposure. In pursuing this, we developed a systematic approach to the mining of a complex biomarker database. This approach helped us find interpretable linear models that could explain a substantial proportion of the variability for all four of the biomarkers of potential harm (WBC, EPI8, DEH11, HDL). These regression models summarize the information originally contained in 169 variables.

We began by applying a general purpose data mining procedure (Random Forest) to find a list of important variables that could plausibly affect the chosen dependant variables and black box models that relate these 


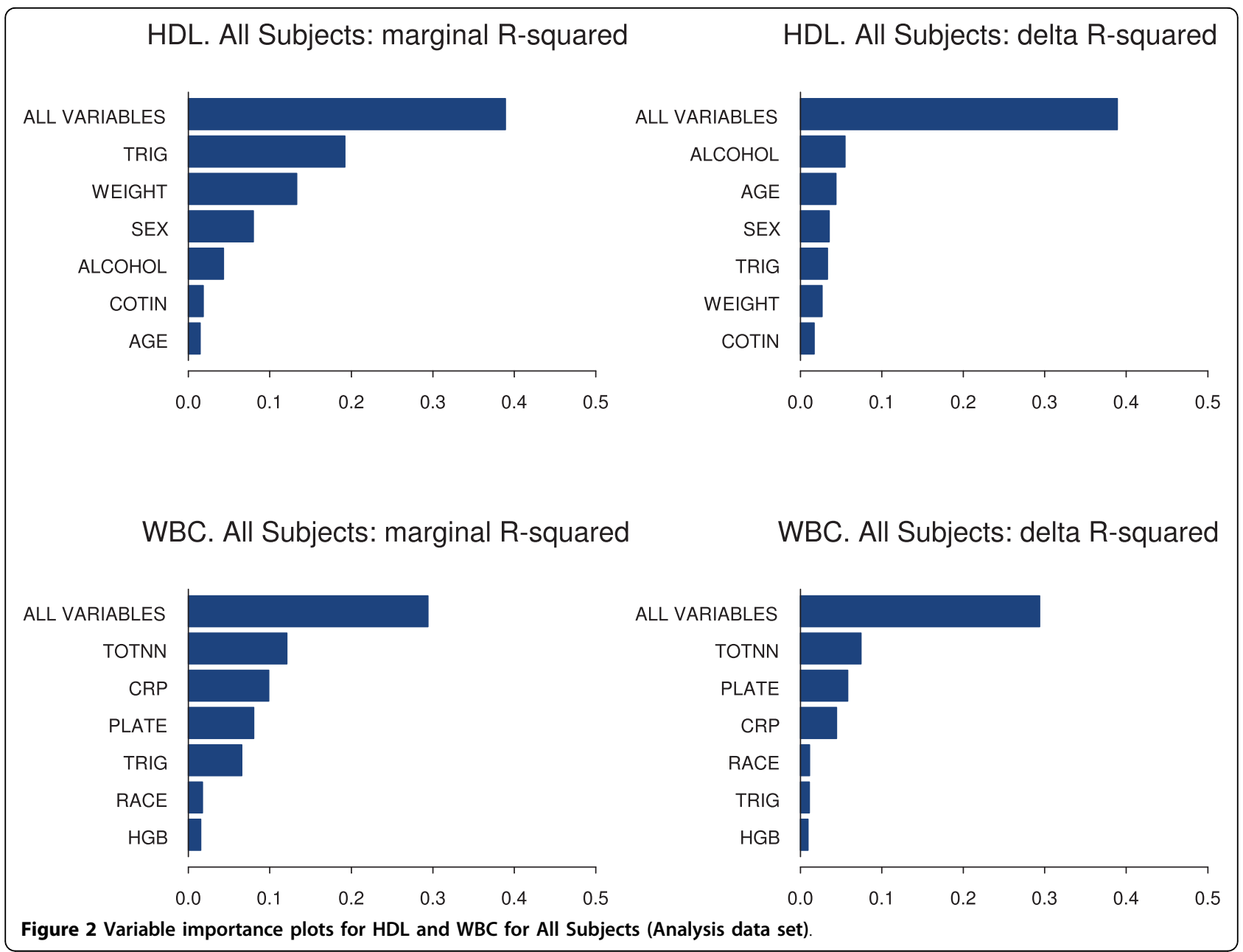

Table 5 Summary of model fit statistics across models for all subjects

\begin{tabular}{lccccc}
\hline Model & $\begin{array}{c}\text { Random Forest R } \mathbf{R}^{\mathbf{2}} \text { : } \\
\text { Analysis Data Set }\end{array}$ & $\begin{array}{c}\text { MARS GCV R } \mathbf{2}^{\mathbf{2}} \\
\text { Analysis Data Set }\end{array}$ & $\begin{array}{c}\text { Linear Regression } \mathbf{R}^{\mathbf{2}} \text { : } \\
\text { Analysis Data Set }\end{array}$ & $\begin{array}{c}\text { Linear Regression } \mathbf{R}^{\mathbf{2}} \text { : } \\
\text { Validation Data Set (applied) }\end{array}$ & $\begin{array}{c}\text { Linear Regression } \mathbf{R}^{\mathbf{2}} \text { : } \\
\text { Validation Data Set (Refit) }\end{array}$ \\
\hline WBC & 0.28 & 0.27 & 0.29 & 0.29 & 0.31 \\
\hline $\mathrm{EPI8}$ & 0.40 & 0.42 & 0.41 & 0.35 & 0.38 \\
\hline $\mathrm{DEH} 11$ & 0.28 & 0.28 & 0.29 & 0.25 & 0.27 \\
\hline $\mathrm{HDL}$ & 0.40 & 0.37 & 0.39 & 0.40 & 0.41 \\
\hline
\end{tabular}

variables to the four BOPH. Results from the Random Forest procedure were used as a starting point for the MARS algorithm which produced the multiple regression models. The regression models found by MARS were then applied and refit to a validation data set in SPLUS. It is remarkable that (as shown in Table 5) the R-squared statistics generated by the Random Forests, MARS and regression procedures are in good agreement with each other, suggesting that the proposed models will be reproducible in future studies.

MARS is a nonparametric regression model in which no assumption is made regarding the function relationship between dependent and independent variables [22,29]. Instead of residual plots, other approaches are commonly used for assessing nonparametric regression models [29]. Due to the availability of a relatively large sample size in our dataset, we set aside a validation dataset of $20 \%$ of the observations and use it to assess the performance of our models. As presented in Table 5 of our manuscript, the R-squared values of the final models based on the analysis dataset are in good agreement with those based on the validation dataset. This approach confirmed the appropriateness of the models. 
The proposed regression models, which involve the transformations of some predictor variables, provide predictive capability comparable to that produced by the Random Forest models. Interactions between dependent variables are not needed. In spite of the generally good agreement of model fit statistics between analysis and validation data sets, the linear models fit to the analysis and validation data sets are not identical, i.e. some variables that are highly significant in the analysis data set do not remain so in the validation data set. These discrepancies, which may suggest aspects of the modeling that will not be generalized to future studies or may be artifacts of the smaller sample size of the validation data set, will be discussed below.

The role of smoking status in our analysis is of special interest. It is notable that smoking status does not appear in any of the regression models. This suggests that the effect of smoking on each biomarker of potential harm is mediated by one or more of the biomarkers of exposure or covariates. 19 additional variables, which measured specifics of smoking behavior, were also entered as candidate variables, but none were included in the final model.

Each model contains at least one BOE predictor: COTIN appears as a predictor of DEH11 and HDL, TOTNN appears as a predictor of EPI8 and WBC, and OHP appears as a predictor of EPI8.

The inclusion of the variables COTIN or TOTNN, instead of smoking status or nicotine equivalents, in the final model for DEH11 or EPI8 does not necessarily mean that these variables by themselves are more biologically important than smoking status or nicotine equivalents. In MARS modeling, the inclusion of a new predictor is dependent on the number of predictors already in the model and the correlation(s) of the new predictor with the remaining predictor(s). COTIN or TOTNN is included in the final model because its contribution and that from other existing predictors accounts for a larger variability in DEH11 and EPI8 than smoking status or nicotine equivalents. On the other hand, the contribution of smoking status or nicotine equivalents is most likely already captured by COTIN or TOTNN and other variables in the model.

As shown in Figure 1, UCRCAL and CRCL are overall important predictors of DEH11, accounting for more variation in DEH11 than the BOE COTIN. Similarly, CRCL is the most important predictor of EPI8, with TOTNN and OHP also playing important roles. The importance of CRCL here is likely related to the fact that DEH11 and EPI8 were both measured in urine. As defined here, CRCL is a marker of kidney function. UCRCAL reflects variability in creatinine production, which is known to be related to muscle mass and is thus influenced by AGE, WEIGHT, and SEX. One of the purposes of this analysis was to investigate various sources of variability, therefore we included the creatinine excretion and creatinine clearance in the model, so that we could understand the magnitude of impact of these factors on the variability. Indeed it was not surprising that, based on the marginal $\mathrm{r}$-squared values (Figure 1), urine creatinine/creatinine clearance had a relatively large contribution on the final models for EPI8 and DEH11. This suggests that normalization for creatinine could potentially reduce the variability in these biomarkers.

The variables TRIG, SEX, WEIGHTK and ALCOHOL CONSUMPTION are all more important predictors of HDL than is the BOE COTIN. The situation is somewhat different for WBC, where the BOE TOTNN appears as the most important predictor, followed by the variables PLATE and CRP.

The model for WBC contains the following predictors: TOTNN, CRP, PLATE, HGB, TRIG, and RACE. The model reflects some well known factors influencing WBC, such as smoking, race and inflammation. The model also indicates a relationship with other hematologic variables such as platelets and red blood cells. Metabolic factors are also known to impact WBC [30].

The model for EPI8 contains the following predictors: TOTNN, OHP, CRCL, AST, WEIGHTK, and VITAMIN. The model suggests that kidney function and body mass are related to the excretion of this biomarker in urine. Exposure to cigarette smoke is thought to increases oxidative stress [11-13], whereas use of vitamin consumption decreases oxidative stress. Oxidative stress may also impact cell membranes, so that enzymes may leak to a higher degree from cells. The weak relationship between AST and EPI8 may be suggestive of the oxidative damage to the cells, since serum AST is localized in heart, brain, skeletal muscle and liver tissue and is generally considered a general biomarker of leaky/damaged cells e.g. hepatocytes as well as myocytes [31]. However this relationship should be interpreted with caution since the contribution of the AST levels to the variability of EPI8 was relatively small (Figure 1). Furthermore AST levels are also reported to be influence by the use of medications [31-33].

The model for DEH11 contains the following predictors: COTIN, UCRCAL, CRCL, AST, ALKPH, NSAID, and VITAMIN. The model suggests that kidney function and muscle mass are related to the excretion of this biomarker in urine. Exposure to cigarette smoke increases platelet activation, whereas use of non-steroidal anti-inflammatory drugs, e.g. aspirin and vitamin consumption decrease platelet activation $[11,15]$. The relationship with serum enzymes is less clear but might indicate that processes, which activate platelets, impact cell membranes, so that enzymes may leak to a higher degree from cells. 
The model for HDL contains COTIN, TRIG, WEIGHTK, AGE, SEX, and ALCOHOL CONSUMPTION. It suggests that bodyweight and male gender impact HDL negatively, whereas older age, female sex and regular alcohol consumption have a positive effect on HDL cholesterol. Higher triglycerides is generally associated with lower HDL cholesterol [34].

\section{Conclusions}

In summary, levels of WBC, EPI8, DEH11 and HDL were statistically significantly related to biomarkers of exposure to cigarette smoking, demographics and life style factors. The statistical models successfully captured a large amount of variability in the biomarkers and depicted the important biological relationships between the biomarker and the effects. Considering the numerous potential sources of variability for the 4 biomarkers and their complex relationships, the R-squared values of $29 \%$ to $41 \%$ are significant.

\section{List of abbreviations}

The abbreviations of all model variables are listed in Table 2.

\section{Acknowledgements}

We thank Lonnie Rimmer, Kim Frost-Pineda, Jianmin Liu and Sunil Kapur for project management and technical assistance with the study.

\section{Author details}

1Pharsight Corporation, a Certara Company, Mountain View, CA 94041-1530, USA. ${ }^{2}$ Altria Client Services Inc, 601 E Jackson Street, Richmond, VA 23219, USA

\section{Authors' contributions}

JHW contributed as the primary modeler. He carried out the statistical modeling and participated in developing the manuscript. QL contributed as the corresponding author of the manuscript. He participated in developing the models and finalized the manuscript. MS participated in developing the manuscript. PEM participated in developing the manuscript. HJR initiated the project and participated in developing the models and the manuscript. All authors read and approved the final manuscript.

\section{Competing interests}

All authors except JHW are or were employees of Philip Morris USA Inc./ Altria Client Services Inc. JHW was an employee of Pharsight Corporation when he was conducting the modeling for this paper. Pharsight Corporation received funding from Philip Morris USA for the modeling.

Received: 21 October 2009 Accepted: 16 March 2010 Published: 16 March 2010

\section{References}

1. US Department of Health and Human Services: The Health Consequences of Smoking: A Report of the Surgeon General. US Department of Health and Human Services, Center for Disease Control and Prevention, National Center for Disease Prevention and Health Promotion, Office on Smoking and Health. Atlanta, GA 2004

2. Ross R: Atherosclerosis: An inflammatory disease. N Eng J Med 1999 340:115-126.

3. Bakhru A, Erlinger T: Smoking cessation and cardiovascular disease risk factors: Results from the Third National Health and Nutrition Examination Survey. PLoS Med 2005, 2:e160.

4. Pryor W, Stone K: Oxidants in cigarette smoke: Radicals, hydrogen peroxide, peroxynitrate, and peroxynitrite. Ann NY Acad Sci 1993, 686:12-28.
5. Miller G, Bauer P, Cooper J, Rosenberg R: Activation of the coagulant pathway in cigarette smokers. Thromb Haemost 1998, 79:549-553.

6. Fusegawa Y, Goto S, Handa S, Kawada T, Ando Y: Platelet spontaneous aggregation in platelet-rich plasma is increased in habitual smokers. Thromb Res 1999, 93:271-278.

7. Craig W, Palomaki G, Haddow J: Cigarette smoking and serum lipid and lipoprotein concentrations: An analysis of published data. BMJ 1989, 298:784-788.

8. FitzGerald G, Oates A, Nowak J: Cigarette smoking and hemostatic function. Am Heart J 1988, 115:267-71.

9. Smith C, Fischer T: Particulate and vapor phase constituents of cigarette mainstream smoke and risk of myocardial infarction. Atherosclerosis 2001, 158:257-267.

10. Abel G, Hays J, Decker P, Groghan G, Kuter D, Rigotti N: Effects of biochemically confirmed smoking cessation on white blood cell count. Mayo Clin Proc 2005, 80:1022-1028.

11. Zedler B, Kinser R, Oey J, et al: Biomarkers of exposure and potentia harm in adult smokers of 3 to $7 \mathrm{mg}$ tar yield (Federal Trade Commission) cigarettes and in adult non-smokers. Biomarkers 2006, 11:201-220.

12. Chehne F, Oguogho A, Lupattelli G, Budinsky A, Palumbo B, Sinzinger $H$ : Increase of isoprostane 8-epi-PGF(2alfa) after restarting smoking. Prostaglandins Leukot and Essent Fatty Acids 2001, 64:307-310.

13. Cracowski J, Durand T, Bessard G: Isoprostanes as a biomarker of lipid peroxidation in humans: Physiology, pharmacology and clinical implications. Trends Phamacol Sci 2001, 23:360-366.

14. Rangemark C, Ciabattoni G, Wennmalm A: Excretion of thromboxane metabolites in healthy women after cessation of smoking. Arterioscler Thromb 1993, 13:777-82

15. Eliasson B, Hjalmarson A, Kruse E, Landfeldt B, Westin A: Effect of smoking reduction and cessation on cardiovascular risk factors. Nicotine \& Tobacco Res 2001, 3:249-255.

16. Roethig H-J, Munjal S, Feng S, Liang Sarkar M, Walk R-AQ, Mendes P: Population Estimates of Biomarkers of Smoke Exposure in U.S. Adult Cigarette Smokers. Nicotine and Tobacco Research 2009, 11(10):1216-25

17. Mendes $\mathrm{P}$, Liang $\mathrm{Q}$, Frost-Pineda K, Munjal S, Walk R, Roethig H: The Relationship between smoking machine derived tar yield and biomarkers of exposure in adult cigarette smokers in the US. Regulatory Toxicology and Pharmacology 2009, 55:17-27.

18. Breiman L: Random Forests. Machine Learning 2001, 45:5-32.

19. Breiman L: Statistical Modeling: The Two Cultures. Statistical Science 2001, 16:199-231.

20. Breiman L, Cutler A, Liu A, Wiener M: RandomForest Package. 2006, Version 4.5-18. $R$ archive.

21. The R Foundation for Statistical Computing: R version 2.5.1. 2007 [http/// www.r-project.org/foundation].

22. Friedman J: Multivariate Adaptive Regression Splines. Annals of Statistics 1991, 19:1-67.

23. Salford Systems: MARS Users Guide. Salford Systems 2001

24. Xu Q, Daszykowski M, Walczak B, Daeyaert F, de Jonge M, Heeres J, Koymans L, Lewi $P$, Vinkers $H$, Janssen P, Massart D: Multivariate adaptive regression splines-studies of HIV reverse transcriptase inhibitors. Chemometrics and Intelligent Laboratory Systems 2004, 72:27-31.

25. Binbing Yu: Approximating the risk score for disease diagnosis using MARS. Journal of Applied Statistics 2009, 36:769-778.

26. Lin H, Wang W, Liu Y, Soong S, York T, Myers L, Hu J: Comparison of multivariate adaptive regression splines and logistic regression in detecting SNP-SNP interactions and their application in prostate cancer. Journal of Human Genetics 2008, 53:802-811.

27. Xiong $\mathrm{R}$, Meullenet J: Application of Multivariate Adaptive Regression Splines (MARS) to the preference mapping of cheese sticks. Journal of Food Science 2004, 69:131-139.

28. Insightful Corp: S-PLUS 7.0 for Windows. Professional Developer 2005

29. Hastie T, Tibshirani R, Friedman J: The elements of statistical learning: data mining, inference, and prediction. Springer 2001.

30. Nakanishi N, Suzuki K, Tatara K: Association between lifestyle and white blood cell count: a study of Japanese male office workers. Occupationa Medicine 2003, 53:135-137.

31. Ozer J, Ratner M, Shaw M, Bailey W, Schomaker S: The current state of serum biomarkers of hepatotoxicity. Toxicology 2008, 245:194-205. 
32. Emerit I, Huang CY, Serejo F, Filipe P, Fernandes A, Costa A, Freitas J, Baptista A, Carneiro de Moura M: Oxidative stress in chronic hepatitis C: a preliminary study on the protective effects of antioxidant flavonoids. Hepatogastroenterology 2005, 52:530-536.

33. Dalla Corte CL, Fachinetto R, Colle D, Pereira RP, Avila DS, Villarinho JG, Wagner C, Pereira ME, Nogueira CW, Soares FA, Rocha JB: Potentially adverse interactions between haloperidol and valerian. Food Chem Toxicol 2008, 46:2369-2375.

34. Miller M, Langenberg P, Havas S: Impact of lowering triglycerides on raising HDL-C in hypertriglyceridemic and non-hypertriglyceridemic subjects. International Journal of Cardiology 2007, 119:192-195.

\section{Pre-publication history}

The pre-publication history for this paper can be accessed here: http://www. biomedcentral.com/1471-2288/10/19/prepub

doi:10.1186/1471-2288-10-19

Cite this article as: Warner et al: Adaptive regression modeling of biomarkers of potential harm in a population of U.S. adult cigarette smokers and nonsmokers. BMC Medical Research Methodology 2010 10:19.

\section{Submit your next manuscript to BioMed Central} and take full advantage of:

- Convenient online submission

- Thorough peer review

- No space constraints or color figure charges

- Immediate publication on acceptance

- Inclusion in PubMed, CAS, Scopus and Google Scholar

- Research which is freely available for redistribution

Submit your manuscript at www.biomedcentral.com/submit 